\title{
THE IMPLEMENTATION OF DISAGGREGATION ALGORITHM IN THE ANALYSIS OF ENERGY CONSUMPTION BASED ON THE INTERNET-OF- THINGS TECHNOLOGY
}

\author{
Husein $^{1}$, Aditya Alviori², Maman Budiman ${ }^{3 *}$, Mitra Djamal ${ }^{4}$ \\ *E-mail: maman@ fi.itb.ac.id
}

\begin{abstract}
The effort to reduce the mass energy usage without involvement of consumers is not effective. Thereby, creating a pathway for anyone of consumer to be much more involved in the energy-saving effort. The implementation of disaggregation algorithm in the analysis of energy consumption is to recognize when and which appliance has the largest energy consumption and being able to control the state of all appliances from anywhere. In this research, the principle of disaggregation is event-based and low-sampling data frequency. A $\mathrm{KWH}-\mathrm{meter}$ is used to send power data to the cloud server via MQTT protocol. The cloud server gathers the energy-consumption data, analyses them and then disaggregates them. The output of the disaggregation algorithm would tell the state (on/off), the average power and the percentage of energy consumed by each appliances. The output will then be sent from the cloud server to Android Apps via MQTT protocol. Then the consumer can easily access and control the energy consumption from their smartphone after knowing it through the disaggregation algorithm.
\end{abstract}

Keywords: Disaggregation, Conservation, Energy Consumption, Energy Analysis, MQTT

\section{INTRODUCTION}

The suggestion of the government in reducing the level of energy consumption in various sectors, has not yet to show maximum results. This failure is caused by several factors, i.e. the lax attitude of consumers and the usage of energy each appliances are not yet measurable in a holistic assessment. Therefore, the disaggregation technique based on the 
internet of things (IoT)

provides technology that underlies the relationship between consumers and power grids so as to motivate and increase the role of consumers towards energy issues. Disaggregation to be done in this study is a process of separation of the total single power consumption into power for every single appliance that are involved in the total power usage. In general, disaggregation better known as the Non-Intrusive Load Monitoring (NILM) was first developed by Hart in 1980. Hart on his research had try to match the real power and reactive power of the tool with which the total power measurement is made at a frequency of $1 \mathrm{~Hz}$ (Hart, 1992). The study goes well, especially on devices with large power. Furthermore, Norford and Leeb had done a research based on methods to detect transient events at a high sampling rate to get appliances with the same power consumption (Norford \& Leeb, 1996). other NILM methods are also developed using Artificial Intelligence (AI). Based on the research, there are two types of technology evolved and it started to be used for disaggregation infrastructure. The idea of this system is to include the manual disaggregation system into the internet of things (IoT) infrastructure. Therefore, operational disaggregation data run time-based data acquisition for disaggregation, namely Low Sampling Frequency and High Sampling Frequency (Iwayemi \& Zou, 2014). Low Sampling Frequency disaggregation acquired data for each 1s - 1 hour. While High Sampling Frequency disaggregation acquired data for each $10 \mathrm{kHz}$ - 1MHz. Disaggregation can also be divided in two categories i.e. Event Based and Non-Event Based. Event Based detects the changing aggregation data related to the on/off of an appliance. While Non-Event Based, work by outlining the aggregate data through the probabilities model and forecasting, such as the HiddenMarkov model (Iwayemi \& Zou, 2014).

Manual disaggregation system is still widely developed today, but the system does not meet the existing challenges such as inaccurate, difficult to access and takes time to analysis the data. With the advent of high number of housing in recent years, manual disaggregation system is not only a waste of time, but also very uncomfortable [1-4]. Recently, Internet of Things (IoT) automatically and more efficiently. Training is not required at this disaggregation algorithm. Disaggregation algorithm constructed in this study can also calculate the average power and energy consumed by appliances. Through the energy calculation 
algorithms, disaggregation can calculate the percentage of consumption of each device in each period.

\section{Statistical Concept}

Disaggregation process requires statistical approach. K-Nearest Neighbour Cluster is an algorithm that classifies the data based on the similarity of a data and other data. The similarity can be determined by the Euclidean distance equation (kNearest Neighbour, 2003). Euclidean distance equation shown in equation 1 as below

$$
D(P, Q)=\quad \sqrt{\sum_{i=1}^{n}\left(P_{i}-q_{i}\right)^{2}}
$$

$P(p 1, p 2, \ldots p n)$ is a set of data that will measure the distance with a set of test data $Q$ $(q 1, q 2, \ldots q n)$. In this study, $\mathrm{k}-\mathrm{NN}$ is used for grouping appliances. Power appliances are not always the same every time.

Where; $\sigma:$ Standard deviation, $\mathrm{N}:$ Number of data,

data-x. In this study, standard deviation filters power data from noise to avoid power surge that occurs suddenly and momentarily. Calculation error in the analysis energy consumption is usually due to appearance the power surges.
Sometimes a power consumption can deviate 5W - 10W shortly. Therefore, algorithms are needed to determine the similarity of power data, so that the lapses on an appliance is still readable as the same device and does not go into another device group. The number of nearest neighbours (k) used in the grouping of electronic equipment is 1 . The reason of the value of $k=1$ is that each power data that is to be grouped will go right into a group of test data closest by Euclidean distance.

Furthermore, Standard deviation is a mathematical equation that measures the diversity of a collection of data (Math is Fun Advanced, 2014). Standard deviation equation shown in equation (2) as below:

$$
\bar{\sigma}=\sqrt{\frac{1}{N} \sum_{i=1}^{N}\left(x_{i}-x\right)}
$$

$x_{i}:$ data $\mathrm{i}_{\mathrm{i}}, x:$ average of

\section{Internet of Things (IoT) Infrastructure System}

The Internet of things (IoT) is the internetworking of physical devices, vehicles (also referred to as "connected devices" and "smart devices"), buildings, and other items - embedded with electronics, software, sensors, actuators, and 
network connectivity that enable these objects to collect and exchange data.

There are three major groups in the IoT system shown in the figure 1. First, the sense and control group. This group is used to measure the condition of appliances and

/ OFF controller systems. Second, visualize and response group displays information to the user (user interface).

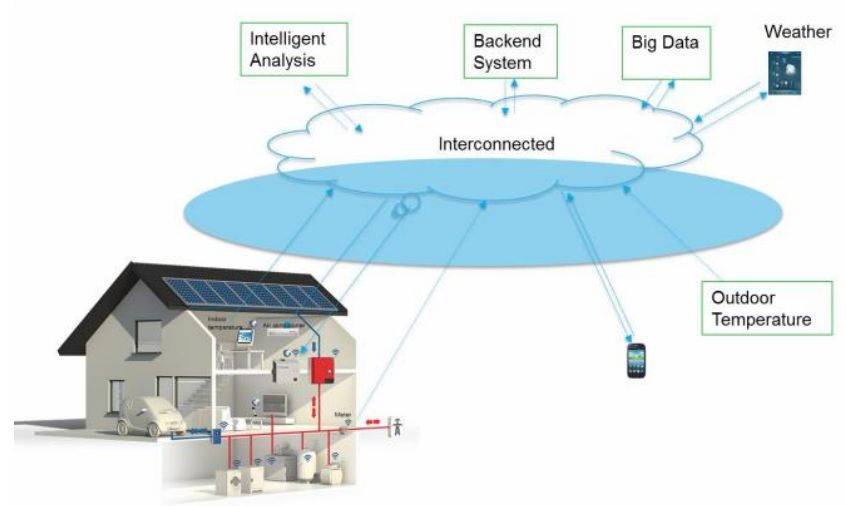

Figure 1. Internet of Thigs (IoT) Infrastrucure for Disaggregation System

PC, smartphone and laptop including a part of this group. Third, intelligent and analysis, big data and backend. Intelligence and analysis process the raw data coming from Kwh meter node for separating single consumption data to be each appliances consumption data. Disaggregation algorithm is injected into intelligent and analysis part. Backend system in this study is using emoncms server to show and save the graph of power data. The three groups are interconnected to each other in the cloud to control the appliances. In this study, the type of sensor according to the function is $\mathrm{KWH}$ meters. The actuator is to switch ON /OFF appliances automation nodes. Automation node consists of embedded ON 


\section{Flow chart of the Disaggregation Algorithm}

The disaggregation algorithm flow chart in general is shown in figure 2 that consist of 11 steps. Firstly, power consumption data from $\mathrm{KWH}$ meter is subscribed by Intelligent and analysis group via MQTT protocol. Secondly, filtering data by using deviation standard equation (2). The number of power data used for filtering are three data which are taken them three times respectively. So that the data can be categorized in a uniform manner, then the value of the standard deviation should be $<3$. 


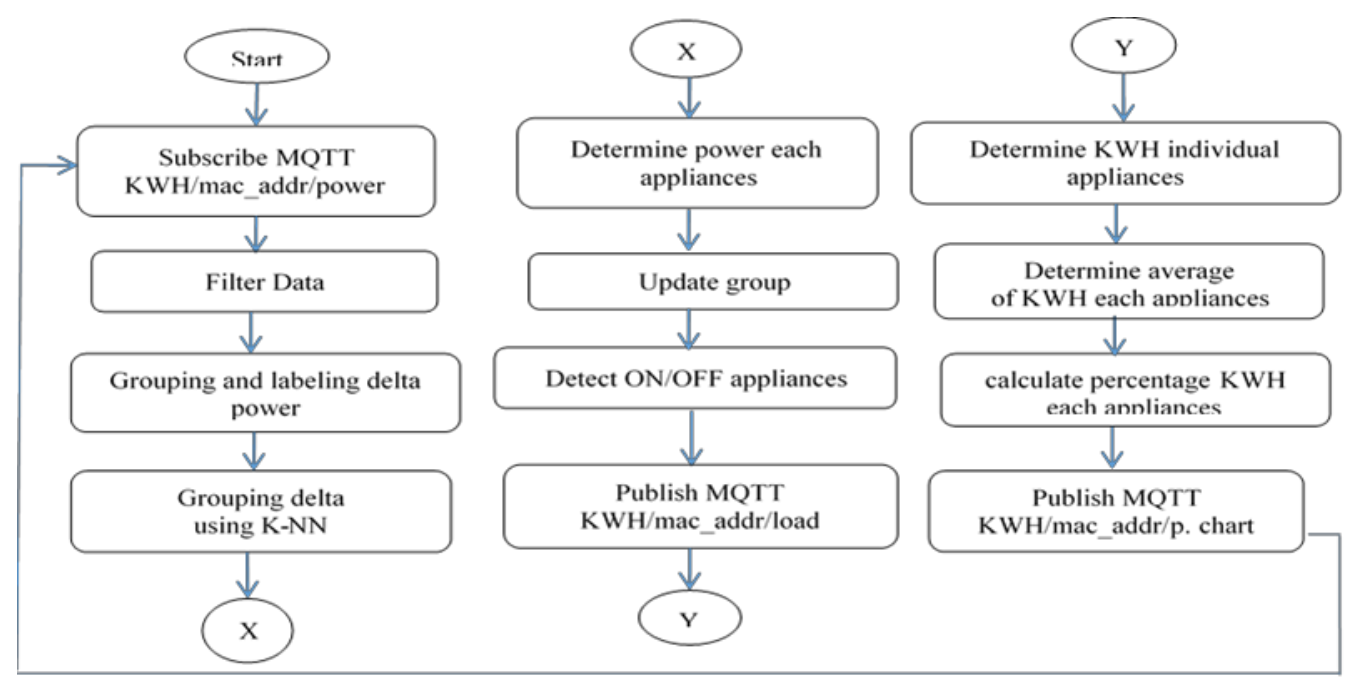

Figure 2. Flow chart of the disaggregation algorithm

Thirdly, Grouping and labeling change of power consumption data. Fourthly, Classification change of power data $(\Delta \mathrm{P})$. The absolute value of $\Delta \mathrm{P}$ will be clustered using k-NN. Fifthly, Calculation of average power consumption for each appliances using equation (3)

$$
\overline{p o w e r}_{\text {label }}=\frac{\sum_{i}^{N} p_{i} \text { ower }}{N}
$$

Sixthly, Update of Group. Average power is used as a reference new "Group". Seventhly, ON/OFF detection. An appliance is stated in the $\mathrm{ON}$ state when the $\Delta \mathrm{P}>$ tolerance. When the condition of the appliance is $\mathrm{ON}$, status and the average power of appliance are sent immediately via MQTT. System take a time when such an appliance in the ON state. Furthermore, intelligent and analysis sent 1 as a sign that appliance is $\mathrm{ON}$. The value 1 is stored in an array. If $\Delta \mathrm{P}<$ tolerance, intelligent and analysis sent 2 as a sign that appliance is OFF. This value also is stored in the same array with an array $\mathrm{ON}$ state. When the number of members in the array rich $3(1+2)$, intelligent and analysis dispatches status and average power consumption of appliance via MQTT protocol. If the process is completed, the array store returned to the empty status. Eighthly, Calculate Energy Consumption (Kwh) of each 
Appliances. If state ON/OFF is experienced by an appliance, calculation of energy consumption can be done using electrical energy equation (4).

$E_{\text {label }}=$ Power label $\times \Delta t_{\text {label }}$

(4)

Power consumption is derived from the average power of each appliance. Time of used come from the time interval when an appliance

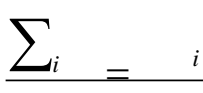

(5)

Average energy is used as variable to calculate the percentage of energy consumption for is $\mathrm{OFF}$ and $\mathrm{ON}$ state. Ninthly, Calculate Average Energy consumption (Kwh) of every single Appliance. After the dictionary of energy has been formed, each label of the dictionary has a collection of energy consumption. The average energy, can be done using equation of average power. Furthermore, the average energy equation is shown in equation (5).

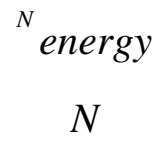

every single appliance. Tenthly, Calculate the percentage of $\mathrm{kWh}$ for every single appliance from the aggregation data. Percentage of the

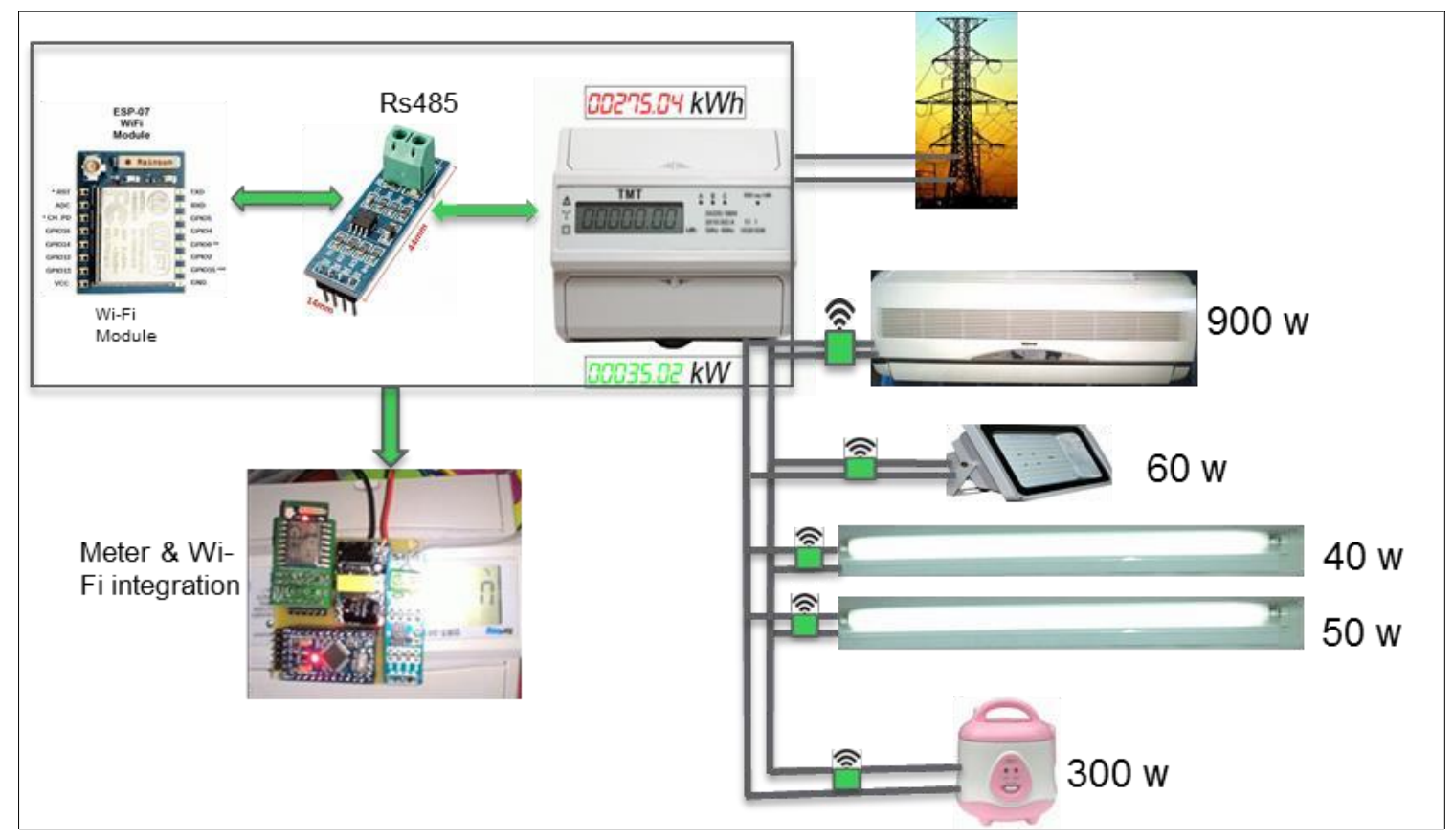

Figure 5. Equipment testing of disaggregation algorithm 
Test performed by random switch $\mathrm{ON}$ and $\mathrm{OFF}$ appliances. The output of Intelligent and analysis group result in a graph and separation power and energy consumption for every single appliances as shown in the figure 6. Figures 6 shows the change of power consumption caused by the changing state (ON/OFF) of the appliances. According to the pie chart above the LED lamp has the longest time of use among appliances but it has the lower percentage of energy consumption about $5 \%$. However, Air conditioner has the short time of use among them, but it has the higher percentage of energy consumption almost $50 \%$.

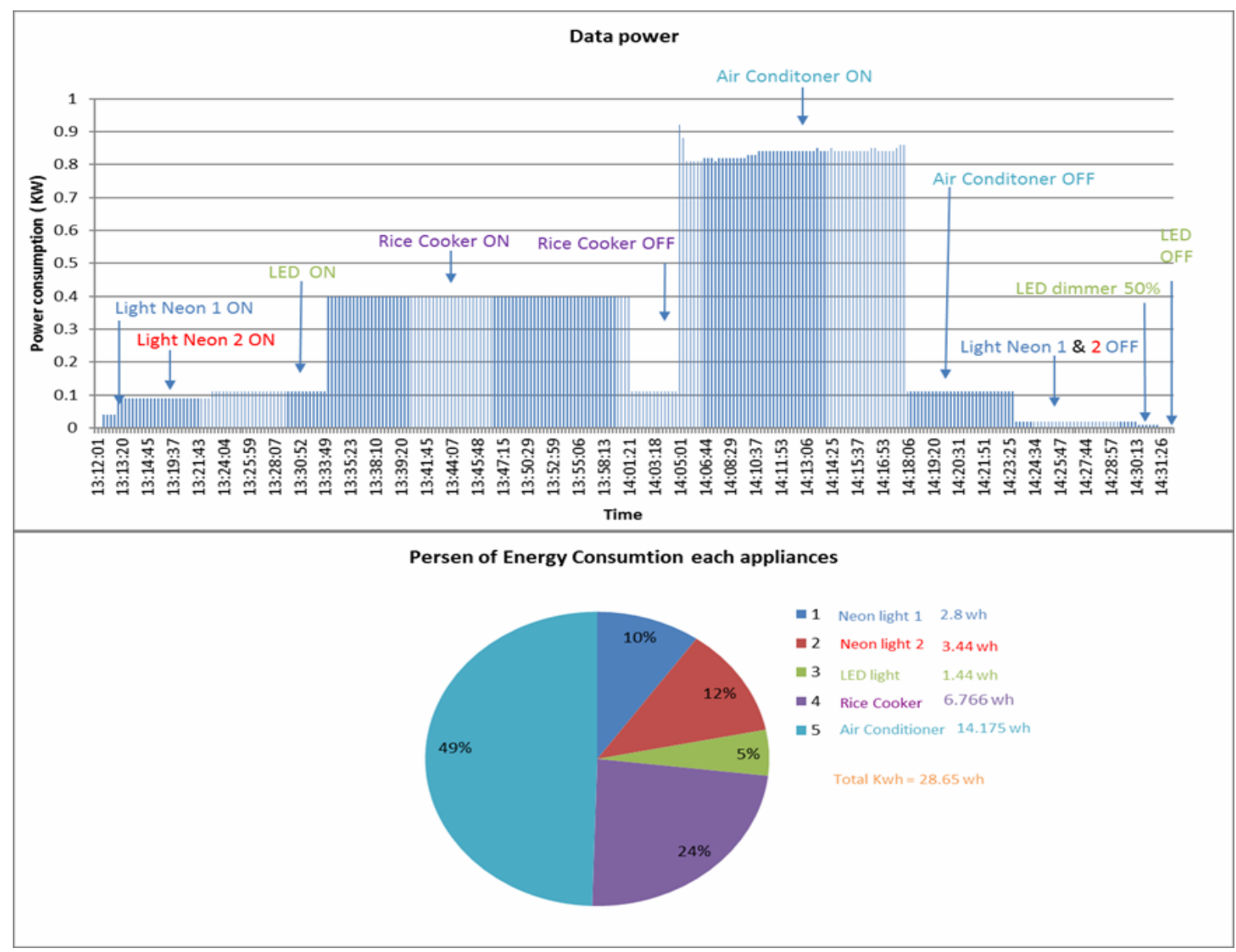

Figure 6. The results test of disaggregation algorithm

The other graph of testing disaggregation algorithm shows a shape similar to the graph of emoncms. The output graph of disaggregation algorithm runs bit late in detecting change little power. It is caused by the filter process take about a minute. 


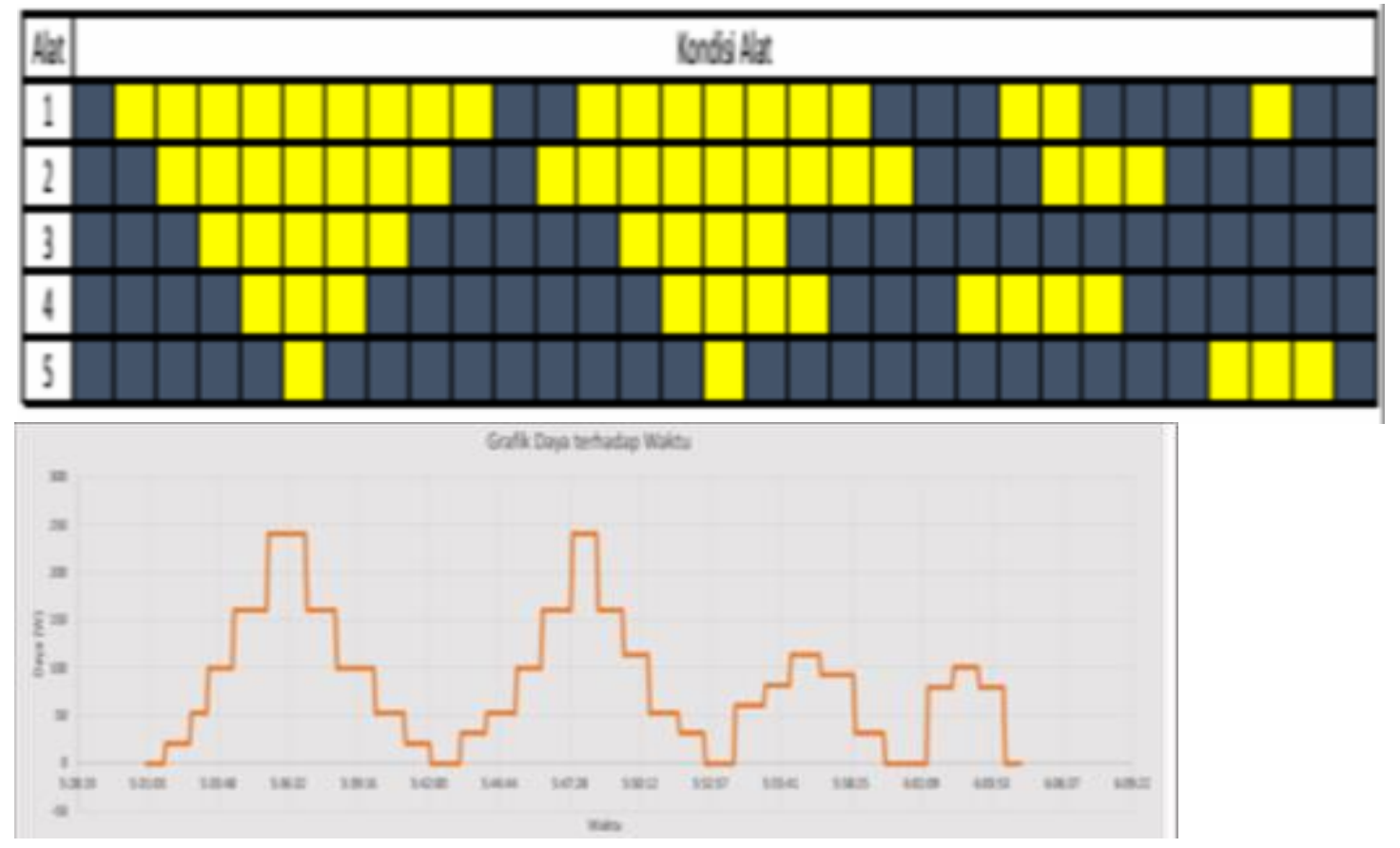

Figure 7. Profile graph of the disaggregation algorithm testing

Figure 7 describes a table contains the state ON/OFF among appliances during the testing time. The yellow color represents ON state of appliances while the gray declares that appliances in the OFF state. The output test of disaggregation algorithms can be subscribed and displayed it in android application via MQTT protocol that shown in Figure 8. Android apps displays energy consumption, state $\mathrm{ON} / \mathrm{OFF}$, average power and the percentage of energy consumption for every single appliance. The Android app always receive change power data and the percentage energy consumption of every single appliance. This shows that the disaggregation algorithm not only succeed in doing disaggregation, but also able to send data to the Android app. Android app successfully receive the information which is displayed to the user interface in the form of a pie chart, table machine of ON/OFF appliances status and average power each appliances. 


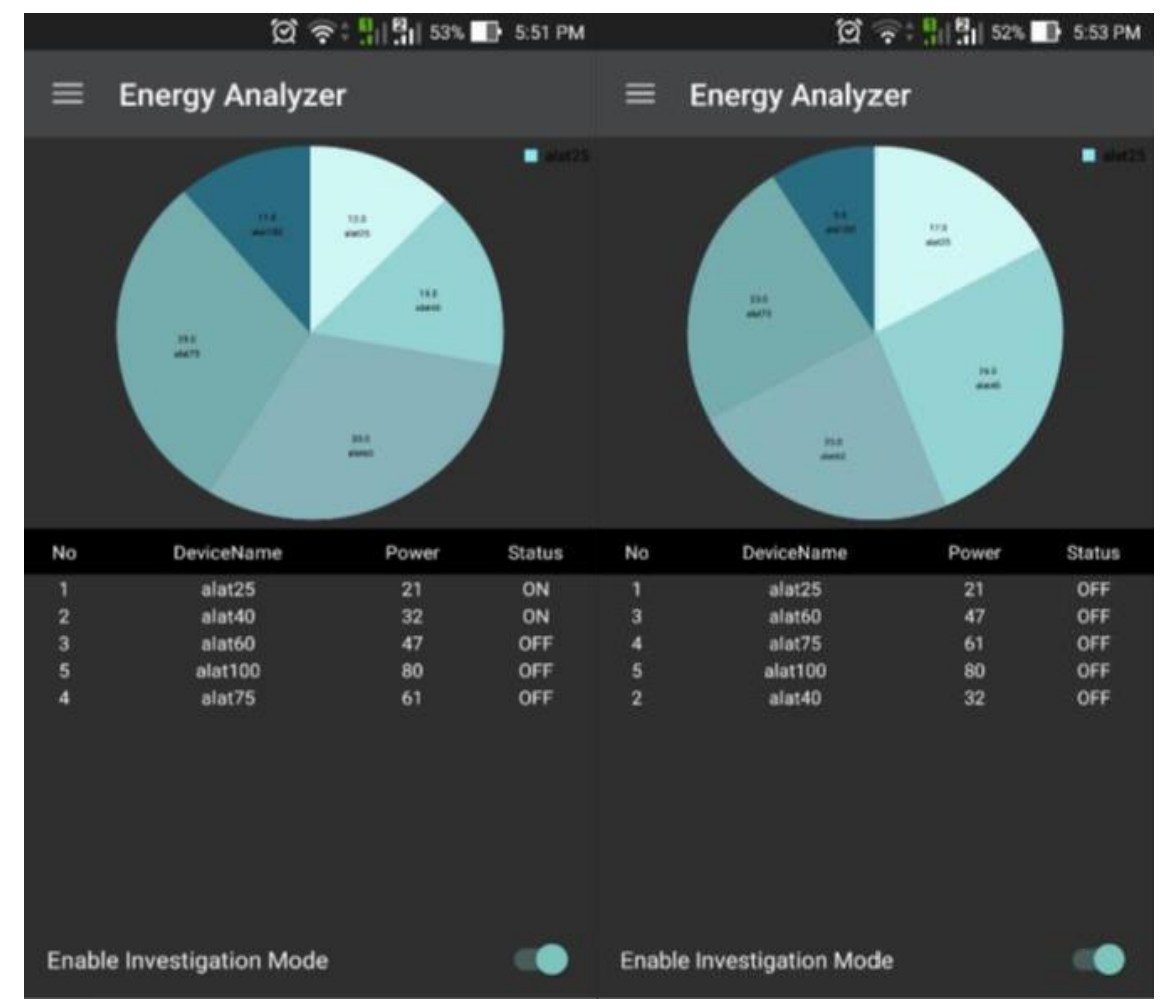

Figure 8 Profile output disaggregation algorithm on the Android app

\section{CONCLUSIONS AND RECOMMENDATIONS}

Implementation of disaggregation algorithm in the analysis energy consumption based on the Internet of Things Technology has been successfully. Information data concerning ON/OFF every single appliance, the average power, and the percentage of energy consumption each appliance displayed them properly. Infrastructure Internet of Things as backbone of this study is running excellently. The suggestions for next research about implementation of disaggregation algorithm is that the parameters input can be added with reactive power to reduce errors in detecting the condition of an appliance.

\section{REFERENCES}


[1] Armel, K. C., Gupta, A., Shrimali, G., \& Albert, A. 2012. Is Disaggregation the Holy Grail of Energy Efficiency?

[2] Capehart, B. L., Turner, W., \& J. Kennedy, W. 2012. Guide to Energy Management. USA: The Fairmont Press, Inc.

[3] Chamberlin, B. 2014. ibmcai. Retrieved from https://ibmcai.com/2014/07/23/building-asmarter-planet-through-an- intelligentinternet-of-things/ Chapter 11 NonParametric Techniques. (2016, Jun

[4] Chapter5-Dictionaries and Structuring Data. (n.d.).

Retrieved from https://automatetheboringstuff .com/chapter5

[14]k-Nearest Neighbor 2003. Retrieved from http://archive2.cra.org/Activities/craw_archiv e/dmp/awards/2003/Mower/K NN.html

[16] Kolter, Z., Batra, S., \& Ng, A. 2011. Energy Disaggregation via Discriminative Sparse Coding. IEEE Trans. Consumer Electronics.

[17] Math is Fun Advanced.

[18] MQTT Essentials: Part 1 - Introducing MQTT. (n.d.). Retrieved from http://www.hivemq.com /blog/mqtt-essentialspart-1-introducing-mqtt
[6] Client-server model.

(n.d.).

[7] Cloud Server. (n.d.).

[8] Comparing Python to Other (2016, Jun

[9] Dictionary.com.

[11] Gupta, A., \& Chakravaty, P. 2012. Impact of Energy Disaggregation on Consumer Behavior.

[12] Hart, G. 1992 Nonjntrusive Appliance Load 28)
Monitoring., (p. $80(12))$. [13] Iwayemi, A., \& Zou, C. 2014. Leveraging Smart Meters for Residential Energy Disaggregation.

[19] Norford, L., \& Leeb, S. 1996. Non-Intrusive Load Monitoring in Commercial Buildings Based on SteadyState and Transient LoadDetection Algorithms. Energy and Buildings. PCMag. (n.d.).

[21] Powers, B., \& Margossian, B. 1991. Using a Rule-Based A914. 1 Rerithm Retriełed rom from http://www.mathsisfun End-Use Load Profiles from Premise-Level Data. Trans IEEE Computer Applications in Power.

[22] Prudenzi, A. 2002. A Neuron Sets Based Procedure for Identifying Domestic 
Appliances Pattern-of-Use

from Energy Recordings at

Meter Panel. IEEE Power

Engineering Society Winter

Meeting.

[25] Techworld. 2015. Retrieved from

http://www.techworld.com/bi g- data/what-is-internet-of-

things-3631109/

[27] Weiss, M., Helfenstein, A., Mattern, F., \& Staake, T. 2012. Leveraging Smart Meter Data to Recognize Home Appliances. 
Jurnal Agribisnis, Vol. 13, No. 2, Juni 2019, [ 18 - 30 ]

ISSN : 1979-0058 